ARTICLE

DOI: $10.1038 / s 41467-018-04472-6$

\title{
Activity of acetyltransferase toxins involved in Salmonella persister formation during macrophage infection
}

Julian A. Rycroft ${ }^{1}$, Bridget Gollan¹, Grzegorz J. Grabe1, Alexander Hall', Angela M. Cheverton', Gerald Larrouy-Maumus ${ }^{2}$, Stephen A. Hare ${ }^{3,4}$ \& Sophie Helaine (iD ${ }^{1}$

Non-typhoidal Salmonella strains are responsible for invasive infections associated with high mortality and recurrence in sub-Saharan Africa, and there is strong evidence for clonal relapse following antibiotic treatment. Persisters are non-growing bacteria that are thought to be responsible for the recalcitrance of many infections to antibiotics. Toxin-antitoxin systems are stress-responsive elements that are important for Salmonella persister formation, specifically during infection. Here, we report the analysis of persister formation of clinical invasive strains of Salmonella Typhimurium and Enteritidis in human primary macrophages. We show that all the invasive clinical isolates of both serovars that we tested produce high levels of persisters following internalization by human macrophages. Our genome comparison reveals that $S$. Enteritidis and S. Typhimurium strains contain three acetyltransferase toxins that we characterize structurally and functionally. We show that all induce the persister state by inhibiting translation through acetylation of aminoacyl-tRNAs. However, they differ in their potency and target partially different subsets of aminoacyl-tRNAs, potentially accounting for their non-redundant effect.

\footnotetext{
${ }^{1}$ Section of Microbiology, Medical Research Council Centre for Molecular Bacteriology and Infection, Imperial College London, Armstrong Road, London SW7 2AZ, UK. ${ }^{2}$ Department of Life Sciences, Medical Research Council Centre for Molecular Bacteriology and Infection, Imperial College London, Armstrong Road, London SW7 2AZ, UK. ${ }^{3}$ Department of Life Sciences, Imperial College London, Exhibition Road, London SW7 2AZ, UK. ${ }^{4}$ School of Life Sciences, University of Sussex, Brighton BN1 9QG, UK. These authors contributed equally: Julian A. Rycroft, Bridget Gollan. Correspondence and requests for materials should be addressed to S.H. (email: s.helaine@imperial.ac.uk)
} 
$\mathrm{N}$ on-typhoidal Salmonellae (NTS), mostly Salmonella enterica serovars Typhimurium and Enteritidis, cause localized intestinal infection in immunocompetent humans, and invasive non-typhoidal disease (iNTS) in HIVinfected adults (where NTS serovars comprise over a third of all bacteraemias ${ }^{1}$ ) and in young children under 5 years old ${ }^{2-4}$. It is estimated that 3.4 million cases of iNTS occur globally each year ${ }^{5,6}$, primarily in sub-Saharan Africa. Although the global incidence of iNTS is much less than typhoid fever, the associated mortality rate of $20-25 \%$ in immunocompetent individuals can exceed $50 \%$ in $\mathrm{HIV}$-infected patients $3,7,8$. This suggests that iNTS kills three- to fourfold more people than typhoid, with estimated deaths per annum approaching 700,000 5,6 .

Cephalosporins remain the first-line antibiotic treatment in Africa and in Europe, but invasive strains are almost universally resistant to the first- and second-generation options. Increasing reports of resistance to third-generation cephalosporins ${ }^{9}$ have bleak implications. Recurrence of the disease has been observed in $20-43 \%$ of cases, which is attributable to reinfection with distinct bacterial isolates only in a minority of cases $(22 \%)^{10}$. The remaining $78 \%$ represent relapses with the same strain. Although this may result from chronic septic foci following incomplete antibiotic treatment or poor drug penetrance to the infected tissues, there is a growing body of evidence indicating this could be due to growth resuscitation of intracellular persister bacteria which, after a period of transient antibiotic-tolerant growth arrest, resume proliferation resulting in clonal relapse of infection ${ }^{10-14}$

Salmonella Typhimurium 12023/14028 is a commonly used laboratory strain originally obtained from cattle. We reported previously that after phagocytosis of this strain by murine macrophages, a significant proportion of the population ceases to grow and is composed of antibiotic-tolerant persisters ${ }^{11}$. We have found that a repertoire of class II toxin/antitoxin (TA) modules, although having no impact on stochastic persister formation in the laboratory medium, is specifically induced upon uptake of the bacteria by macrophages. In infection conditions, these stress-responsive elements are involved in the formation of intramacrophage persisters in a non-redundant manner ${ }^{11}$. Class II TA operons encode a non-secreted toxin, which inhibits an essential cellular function such as RNA translation or DNA replication, and an antitoxin that interacts with and neutralizes the toxin ${ }^{15}$. The toxin is relatively stable, whereas the antitoxin is labile and degraded under various stress conditions, leading to the build-up of free toxin and growth arrest of the bacterial cell $^{16}$. We recently revealed that one such toxin, TacT, contributes to Salmonella Typhimurium entry into the persister state through acetylation of aminoacyl-tRNA molecules, thereby halting translation ${ }^{17}$.

Here we investigate whether strains of $S$. Typhimurium and $S$. Enteritidis isolated from humans also respond to cellular internalization by a 1000 -fold increase in persister formation. We characterize persister induction in a collection of seven clinical isolates from patients in Malawi and find that all the invasive strains of $S$. Typhimurium and Enteritidis that we tested form persisters to a similar extent following internalization by human macrophages. A genomic comparison reveals that all strains encode three TacT-like toxins amongst a conserved repertoire of TAs. We fully characterize the structure and function of all the related Tac toxins found in $S$. Enteritidis and $S$. Typhimurium. We show that all three toxins induce the persister state by inhibiting translation through acetylation of aminoacyl-tRNAs. However, they differ in their potency and target partially different subsets of aminoacyl-tRNAs, potentially accounting for their non-redundant effect.

\section{Results}

Non-typhoidal Salmonella form persisters in human macrophages. We reported that the formation of persisters in the laboratory reference strain of S. Typhimurium 12023 is dramatically increased after phagocytosis by murine macrophages ${ }^{11}$. Because of the strong evidence for clonal relapse of Salmonella infection following antibiotic treatment ${ }^{10}$, we investigated persister formation of clinical invasive strains of $S$. Typhimurium and Enteritidis (four and three isolates, respectively) in human primary macrophages. All clinical isolates were collected from blood cultures taken from patients in the Queen Elizabeth Hospital, Blantyre in Malawi between 2003-04 (Supplementary Table 1). We compared the behaviour of each clinical isolate to that of the broadly studied reference stains of each serovar. For all clinical and reference isolates, internalization of Salmonella by human macrophages stimulated a similar increase (of approximately 1000 -fold) in the proportion of persisters, compared to those already present in the inoculum (Fig. 1a), with similar proportions being recovered after exposure to gentamicin (all Typhimurium strains: log percentage survival $-1.65 \pm 0.23$ ) or cefotaxime (log percentage survival $-1.19 \pm 0.3)$. Very similar stimulation of persister formation was observed for all the isolates after uptake by macrophage-like cell lines, as evidenced by the biphasic killing curves (Supplementary Fig. 1). This experiment reveals that the formation of antibiotic-tolerant persisters in response to internalization by host cells is a common feature of the Salmonella strains.

Fourteen chromosomal class II TA modules were identified previously in S. Typhimurium 12023 as contributing to persister formation $^{11}$. We compared the repertoire of the class II TA modules in the different clinical isolates. Repertoires are perfectly conserved between the strains of the same serovar, and the comparison between $S$. Typhimurium and $S$. Enteritidis revealed that all $S$. Enteritidis isolates display a one amino acid change in one of the toxins, and that they also lack relBE1 and parDE (Fig. 1b) that are important TA modules for the formation of persisters by $S$. Typhimurium ${ }^{11}$. The polymorphic toxin was an uncharacterized toxin sharing significant amino acid identity (46.8\%) with the previously characterized TacT (Supplementary Fig. 2); we named it TacT2. A third-related toxin, TacT3, showing $28.3 \%$ of the amino acid identity to TacT, is conserved across serovars (Supplementary Fig. 2). Since no significant difference was noticeable in the ability of strains from the two serovars to form persisters (Fig. 1a), we hypothesized that TacT2 $2_{\text {SEn }}$ might compensate for the absence of RelE1 and ParE in persister formation.

Growth arrest induced by the activity of TA toxins stimulates the formation of antibiotic-tolerant persister bacteria. Accordingly, overexpression of $\operatorname{tacT} 2_{S E n}$ or tacT3 and to a lesser extent tacT $2_{S T m}$ into the single deletion mutant of the corresponding TA module led to an increase in the proportion of Salmonella surviving the exposure to bactericidal concentrations of cefotaxime during growth in vitro, without having affected the MIC (Fig. 1c and Supplementary Fig. 3A). This corresponded to an increase in persister proportions, as illustrated by the biphasic killing curves obtained upon exposure to 20 times the MIC, out of the range where survival rate is influenced by the antibiotic dose (Supplementary Fig. 3B). The increase in persister formation induced by overexpression of $t a c T 2$ SEn and tacT3 was fully counteracted by overexpression of their respective antitoxins, tacA2 and tacA3 (Fig. 1c). Together, these results indicate that TacT2 $2_{\text {SEn }}$ promotes persister formation to a greater extent with respect to $\mathrm{TacT} 2_{\mathrm{STm}}$. 
a

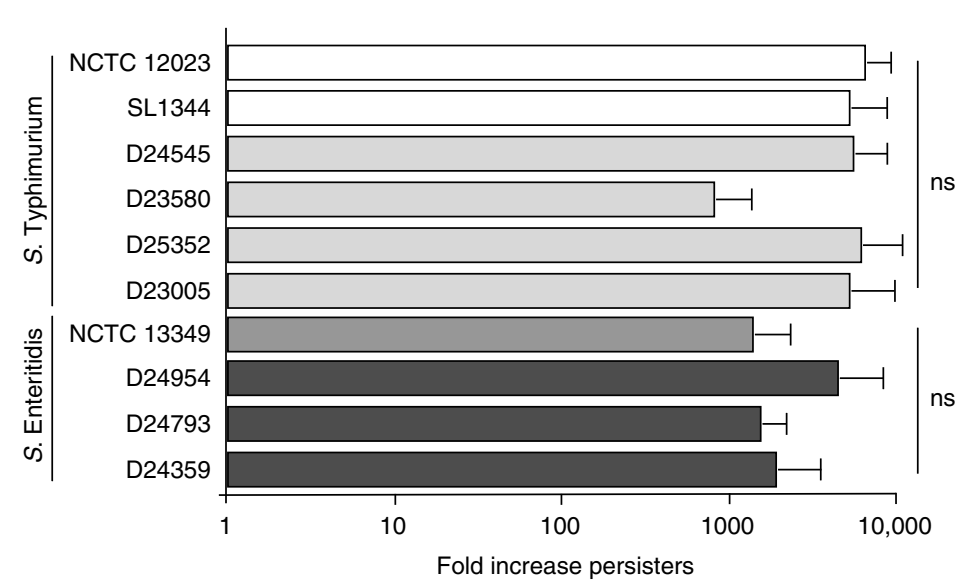

C

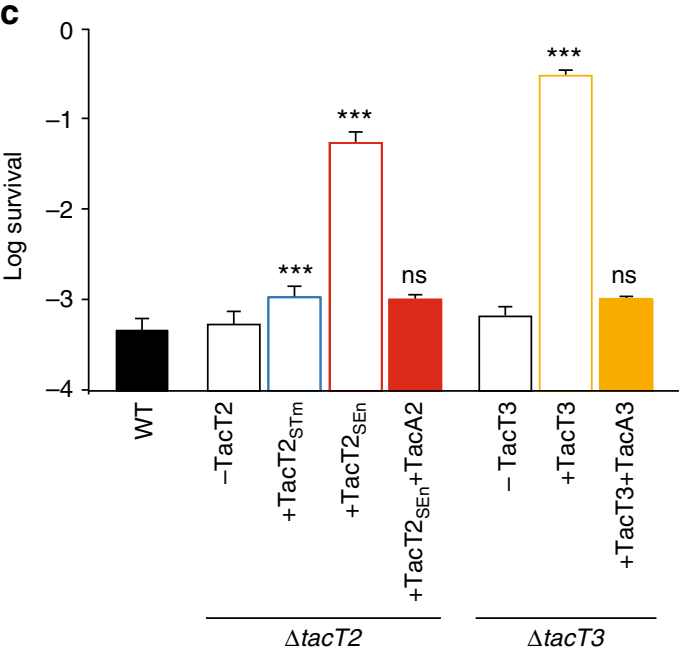

b

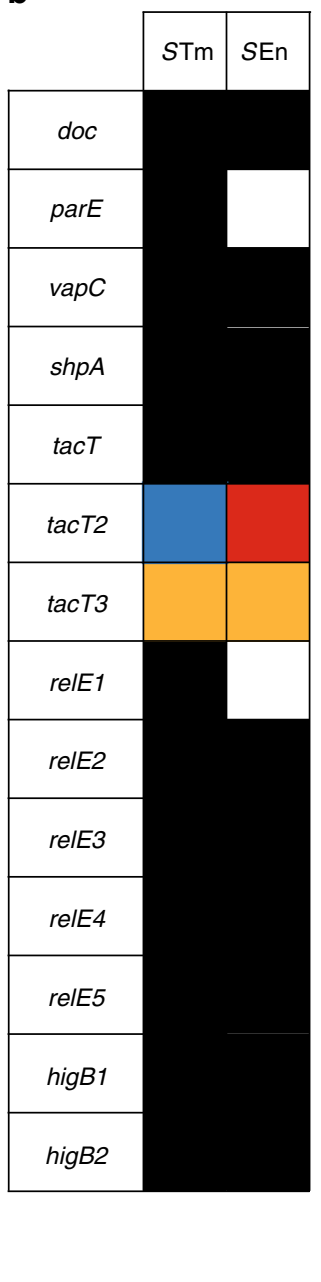

Fig. 1 Non-typhoidal Salmonella strains induce persister formation following internalization by human macrophages. a Fold increase in persisters caused by $30 \mathrm{~min}$ internalization in primary human macrophages relative to levels in inocula in clinical isolates of $S$. Typhimurium (white and light grey bars) and $S$. Enteritidis (dark grey bars), measured after exposure to gentamicin. Data represent the mean \pm SEM $(n \geq 5)$ and were analysed using one-way ANOVA. $\mathbf{b}$ Comparison of the repertoire of class II TA modules between S. Typhimurium and S. Enteritidis serovars. Boxes of same colour-fully conserved; white boxes-absence; different colour boxes-polymorphism. c Proportion of bacteria surviving $4 \mathrm{~h}$ exposure to bactericidal concentrations of cefotaxime in cultures of $\mathrm{S}$. Typhimurium 12023 wild-type, $\triangle$ tacAT2, $\Delta$ tacAT2 pBAD33::tacT2 STm $_{2}, \Delta$ tacAT2 pBAD33::tacT2 SEn, $\Delta$ tacAT2 pBAD33::tacT2 SEn and pCA24N:: tacA2, or $\triangle$ tacAT3, $\triangle$ tacAT3 pBAD33::tacT3, $\triangle$ tacAT3 pBAD33::tacT3 and pCA24N::tacA3. Arabinose and IPTG were added to all cultures in the fresh medium during the lag phase, then antibiotic treatment started $1 \mathrm{~h}$ later. Data represent the mean \pm SEM $(n \geq 3)$ and were analysed using a Student's $t$ test, compared to WT (ns, non-significant; ${ }^{\star \star \star} p<0.005$ )

Acetyltransferases $\mathrm{TacT} 2_{\mathrm{SEn}}$ and TacT3 alter the translation. We previously showed that TacT is a Gcn $5 \mathrm{~N}$-acetyltransferase (GNAT) toxin that controls Salmonella growth through acetylation of aminoacyl-tRNA molecules ${ }^{17}$.

Overexpression of the $\operatorname{tacT} 2_{S T m}$ gene in a deletion mutant of the corresponding TA module had no noticeable effect on $S$. Typhimurium growth, whereas overexpression of $t a c T 2_{S E n}$ extended the lag phase dramatically (Fig. 2a and Supplementary Fig. 4). Overexpression of the tacT3 gene in a deletion mutant of the corresponding TA module dramatically extended the lag phase of S. Typhimurium (Fig. 2a and Supplementary Fig. 4), indicating that $\mathrm{TacT} 2_{\mathrm{SEn}}$ and $\mathrm{TacT} 3$, but not $\mathrm{TacT} 2_{\mathrm{STm}}$ toxins, prolong the bacterial non-growing state. This growth inhibition was counteracted by concomitant overexpression of the corresponding antitoxin $\operatorname{tac} A 2$ or $\operatorname{tac} A 3$ gene (Fig. 2a). These results show that the TacAT2 $2_{\text {SEn }}$ and TacAT3 operons encode functional TA modules.

The toxicity of TacT is dependent on its acetyltransferase activity (the transfer of an acetyl group from acetyl coenzyme A
(Ac-CoA) to the target molecule). Accordingly, for each toxin, a single amino acid substitution of the predicted catalytic site responsible for transfer of the acetyl moiety to the acceptor molecule (Y137F for TacT2 ${ }_{\text {SEn }}$ and Y143F for TacT3) completely abolished the toxicity in Salmonella (Fig. 2b).

The crystal structure of the purified non-toxic $\mathrm{TacT}^{\mathrm{Y} 140 \mathrm{~F}}$ mutant protein revealed an acetyltransferase fold and an AcCoA molecule associated in the active site ${ }^{17}$. We obtained the crystal structure of purified TacT3 $3^{\mathrm{Y} 143 \mathrm{~F}}$ in $1.5 \AA$ resolution (Fig. 2c-d and Supplementary Fig. 5, and Supplementary Table 3). The fold of TacT3 appeared to be that of an acetyltransferase similar to TacT. with the exception of an additional C-terminal alpha-helix (Fig. 2c, right panel). Based on its high identity with TacT (46.8\%), we modelled the structure of TacT2 (Fig. 2d, right panel). Similarly to our results with TacT, purified TacT3 forms a dimer with a comparable dimerization interface (Supplementary Fig. 6). Examination of the surface electrostatic potential of TacT2 and TacT3 revealed a positively charged groove, leading from the active site of one monomer to another patch of positive charge on 

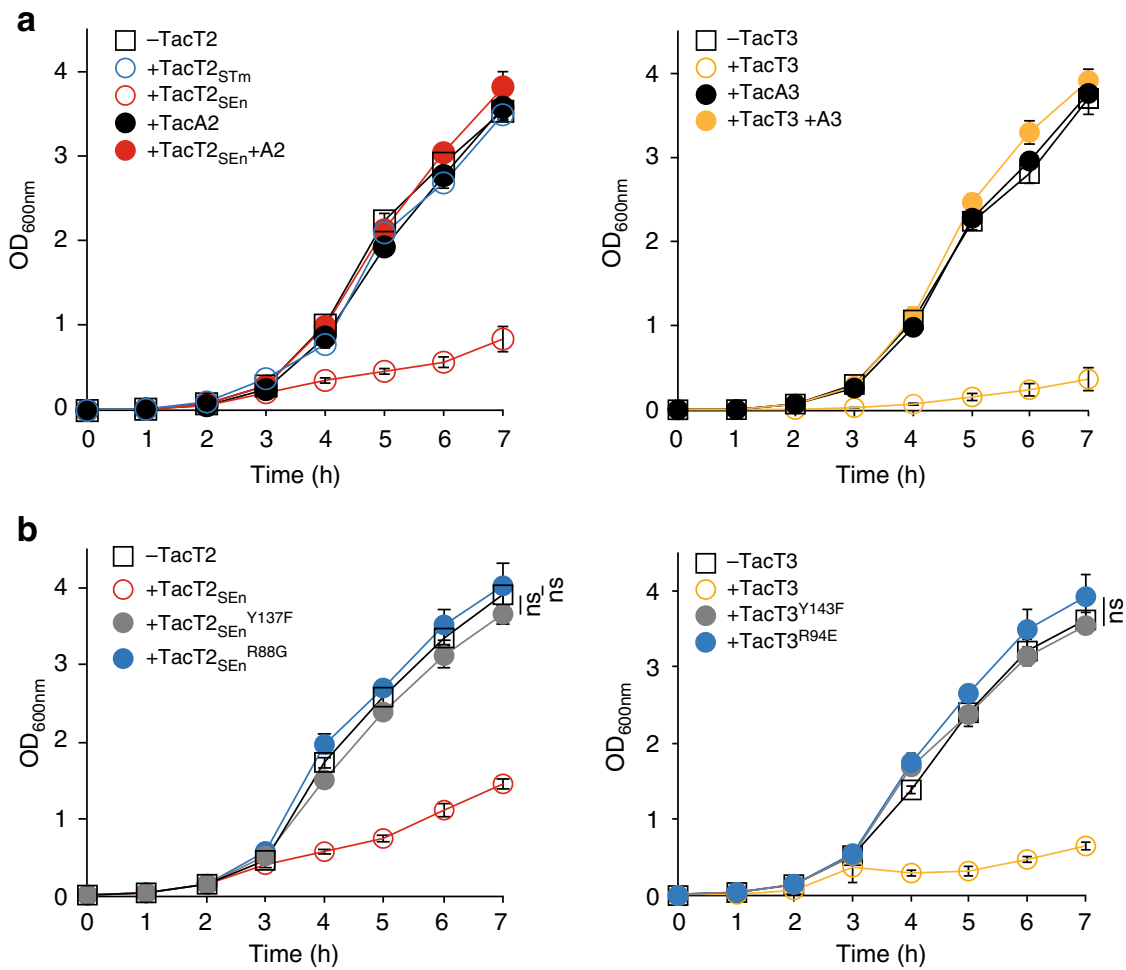

C

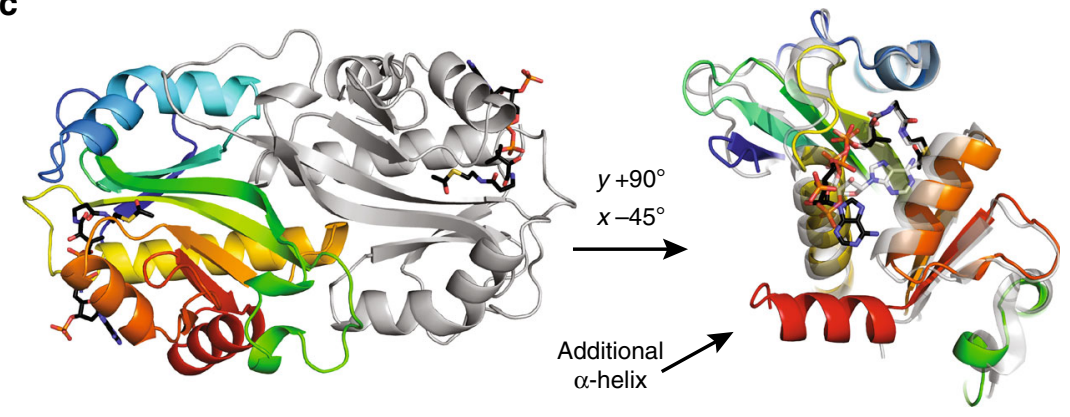

d
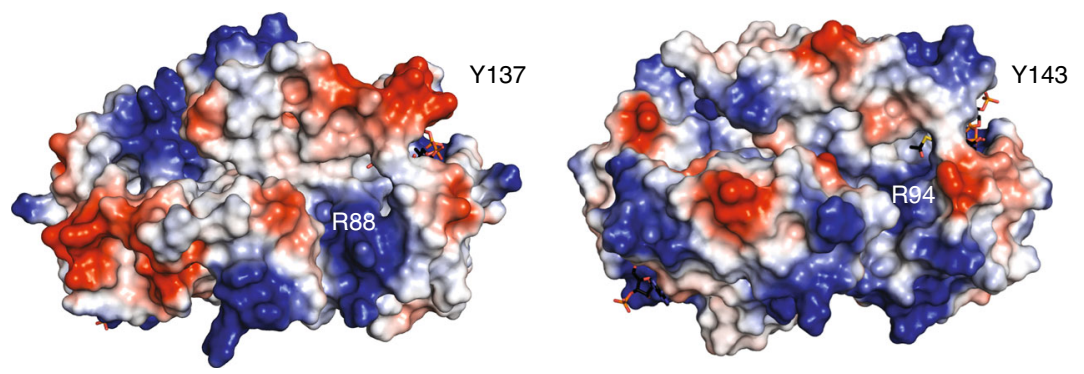

Fig. 2 TacT2 2 SEn and TacT3 are functional acetyltransferase toxins. a Left panel: Growth curves of S. Typhimurium $12023 \triangle$ tacTA2 carrying pBAD33 (-TacT2), pBAD33::tacT2 ${ }_{S T m}$ (+TacT2 $2_{S T m}$ ), pBAD33::tacT2 SEn $_{n}\left(+\right.$ TacT2 $_{\text {SEn }}$ ), pCA24N::TacA2 (+TacA2) or pBAD33::tacT2 $2_{\text {SEn }}$ and pCA24N::tacA2 (+TacT2 SEn + TacA2). Right panel: Growth curves of S. Typhimurium 12023 AtacTA3 carrying pBAD33 (-TacT3), pBAD33::tacT3 (+TacT3), pCA24N:: TacA3 (+TacA3) or pBAD33::tacT3 and pCA24N::tacA3 (+TacT3 + TacA3). All cultures were supplemented with arabinose and IPTG in fresh rich medium during the lag phase and growth was monitored by optical density. b Left panel: Growth curves of S. Typhimurium $12023 \Delta$ tacTA2 expressing from pBAD33, the wild-type S. Enteritidis toxin ( + TacT2 $2_{\text {SEn }}$ ) or point mutant toxins ( + TacT2 ${ }^{\mathrm{Y}_{137 F}}{ }_{\text {SEn }}$ ) or ( + TacT2 ${ }^{\text {R88G }}{ }_{\text {SEn }}$ ), or carrying the empty vector (-TacT2). Right panel: Growth curves of S. Typhimurium 12023 stacTA3 expressing from pBAD33, the wild-type toxin (+TacT3), or point mutant toxins $\left(+\mathrm{TacT}^{\mathrm{Y} 143 \mathrm{~F}}\right)$ or $\left(+\mathrm{TacT}^{\mathrm{R} 94 \mathrm{E}}\right)$, or carrying the empty vector (-TacT3). All cultures were supplemented with arabinose in fresh rich medium during the lag phase. c Left panel: Cartoon representation of the dimeric structure of TacT3 ${ }^{\mathrm{Y} 143 \mathrm{~F}}$. Chain $\mathrm{A}$ is coloured from blue at the $\mathrm{N}$ terminus to red at the $\mathrm{C}$ terminus, and Chain B is coloured light grey. Right panel: Cartoon superimposition of TacT3 ${ }^{\mathrm{Y} 143 \mathrm{~F}}$ (coloured) and TacT $\mathrm{Y140F}$ (grey). TacT3- and TacT-bound Ac-CoA molecules are shown as black and grey sticks, respectively. (PDB: 6G96). d Electrostatic potential of the surface of the modelled TacT2 $2_{\text {SEn }}$ (left panel) or TacT3 (right panel) dimer showing positive potential in blue and negative in red. Residues mentioned in the text are labelled. $\mathbf{a}$, $\mathbf{b}$ Data represent the mean $\pm \operatorname{SEM}(n \geq 3)$ and were analysed using a Student's $t$ test (ns, non-significant) 
the second molecule of the dimer (Fig. 2d) -features also present at the surface of TacT. Single amino acid substitution of R88G and R94E in TacT2 2 SEn and TacT3, respectively, abolished the toxicity in Salmonella, in agreement with our previous data for TacT (Fig. 2b). These results show that, similarly to TacT, TacT $2_{\text {SEn }}$ and TacT3 exhibit positive amino acids at their surface that are essential for their activity.

TacT promotes persister formation through interference with protein synthesis ${ }^{17}$. To determine if TacT $2_{\mathrm{SEn}}$ and TacT3 have the same activity, we measured the rates of translation using pulsechase of radio-labelled methionine ${ }^{18}$. As a positive control, we treated a bacterial culture with chloramphenicol, as this antibiotic inhibits mRNA translation. Upon induction of gene expression of $\operatorname{tacT} 2_{S E n}$ and tacT3, but not of the parE toxin predicted to target DNA, methionine incorporation was immediately decreased (Supplementary Fig. 7), indicating that all active TacT toxins alter translation.

Tac toxins block translation through aminoacyl-tRNA acetylation. We did not detect any noticeable effect of overexpression of $\operatorname{tacT} 2_{S T m}$ on Salmonella growth (Fig. 2a and Supplementary Fig. 4), however, since we observed a mild effect on persister formation (Fig. 1c) and a defect in recovery of macrophageinduced persisters in a single deletion mutant for tacT2 (referred to as ta9 in ref. ${ }^{11}$ ), we considered that $\mathrm{TacT} 2 \mathrm{STm}$ was, nevertheless, a functional acetyltransferase. Each toxin, TacT2 $2_{\mathrm{STm}}$, TacT2 2 Sn and TacT3, was purified by co-expression with their cognate antitoxins to prevent inhibition of the growth of the recombinant $E$. coli strain. The toxins formed stable complexes with their antitoxins, that were later dissociated through denaturation on a nickel column, followed by toxin refolding. We investigated the effect of purified TacT2 $2_{\text {STm }}$, TacT $2_{\text {SEn }}$ and TacT3 in a cell-free expression assay using production of dihydrofolate reductase (DHFR) as a read-out of successful translation. All toxins inhibited production of DHFR when supplemented with Ac-CoA, although to different extents (Fig. 3a).

TacT acetylates the primary amine group of the amino acid on charged tRNA molecules, thereby blocking peptide bond formation and protein synthesis ${ }^{17}$. Similarly, purified TacT2 $2_{\text {SEn }}$, TacT $2_{\mathrm{STm}}$ and TacT3 supplemented with $\left[{ }^{14} \mathrm{C}\right] \mathrm{Ac}-\mathrm{CoA}$ acetylated tRNA molecules in the cell-free expression samples (Fig. 3a) or extracted from $\triangle$ tacATtacAT2tacAT3 deletion Salmonella (Fig. $3 c$ ), as revealed by the autoradiography after acid-urea PAGE. Remarkably, the extent of acetylation of tRNA molecules in the samples mirrored the extent of inhibition of DHFR production (Fig. 3a, bottom panels) and that of toxicity (Fig. 2a and data not shown). TacT3 appeared to be the most potent toxin, and comparison of its active site with that of TacT revealed a difference in the orientation of Ac-CoA (Fig. 3b). The adenine moiety is bent and more engaged in the binding pocket of TacT (and the modelled TacT2) compared to that of TacT3, most likely owing to the bulky side chain of the Trp142 residue present in TacT3. In TacT3, the adenine base of Ac-CoA is further stabilized outside the binding pocket by a hydrogen bond between its amine group and a carbonyl oxygen of the Gly145 residue (Fig. 3b). It is possible that this different conformation allows rapid unloading of coenzyme A after transfer of the acetyl moiety to the target substrate, increasing the processivity of the enzyme.

We previously showed that Peptidyl-tRNA hydrolase (Pth), which is an essential esterase of all bacterial species that recycles free peptidyl-tRNA molecules released during premature termination of translation ${ }^{19}$, detoxified the acetylated charged tRNAs ${ }^{17}$. Therefore, we tested whether the acetylation of TacT2 $2_{\text {SEn }}$, TacT2 $2_{\text {STm }}$ and TacT3-corrupted tRNAs could be resolved by $\mathrm{Pth}$. When tRNA molecules acetylated by the toxins in vitro were subsequently incubated with purified Pth, a strong decrease in the radioactive acetylation signal was detected (Fig. 3c). In agreement, overexpression of $S$. Typhimurium pth, although having a detrimental effect on the bacterial growth, counteracted the effects of TacT2 $2_{\mathrm{SEn}}$ and TacT3 on growth inhibitory or persister induction (Supplementary Fig. 8).

Altogether, these results show that all TacT-like toxins present in Salmonella Typhimurium and Enteritidis block the primary amine group of the amino acid on charged tRNA molecules (Fig. 3d), and that they do this to different extent.

TacT $2_{\text {SEn }}$ acetylates aminoacyl-tRNAs more than TacT $2_{S T m}$ The activity of TacTs relies on several positively charged residues exposed at the surface of the molecule. Interestingly, TacT2 variability between serovars is limited to one amino acid with reversed charge $(\mathrm{E} 29 \mathrm{~K})$, from negative in $\mathrm{TacT} 2_{\mathrm{STm}}$ to positive in TacT $2_{\text {SEn }}$ at the surface of the molecule, in close proximity to the positive groove, leading to the active site (Fig. 4a). We investigated how this single amino acid change has an impact on the toxicity of TacT2.

Since our model proposed that the binding between the toxin and the target aminoacyl-tRNA(s) involves electrostatic bonds and the predicted $\mathrm{pI}$ of the two enzymes differ significantly (7.13 and 6.62 for TacT2 $2_{S E n}$ and $\mathrm{TacT} 2_{\mathrm{STm}}$, respectively), we hypothesized that TacT2 $2_{\mathrm{STm}}$ could work better in acidic than neutral $\mathrm{pH}$. We compared the enzymatic activities of TacT2 $2_{\mathrm{SEn}}$ and $\mathrm{TacT} 2_{\mathrm{STm}}$ over a $\mathrm{pH}$ range from 6.0 to 7.5 . AminoacyltRNAs extracted from $\Delta$ tacATtacAT2tacAT3 Salmonella and treated with purified TacT2 $2_{\text {SEn }}$ supplemented with $\left[{ }^{14} \mathrm{C}\right] \mathrm{Ac}-\mathrm{CoA}$ were acetylated at all $\mathrm{pH}$ conditions tested. However, TacT $2_{\mathrm{STm}}$ only acetylated the aminoacyl-tRNAs efficiently at a $\mathrm{pH}$ of 7.5 (Fig. 4b). This result does not support a model of increased activity at acidic $\mathrm{pH}$, but shows that TacT $2_{\mathrm{STm}}$ is more susceptible to $\mathrm{pH}$ changes than TacT2 $2_{\text {SEn }}$.

Measurement of the thermostability of the two purified enzymes indicated a lowered Tm by at least $4{ }^{\circ} \mathrm{C}$ for TacT2 ${ }_{\mathrm{STm}}$, compared to TacT $2_{\mathrm{SEn}}$ (Supplementary Fig. 9), suggesting that TacT $2_{\mathrm{STm}}$ is a less stably folded protein. The model of the structure of TacT2 2 SEn suggests the presence of stabilizing hydrogen bonds formed between the side chain of K29 and the two neighbouring residues Phe17 and Tyr15 that cannot be formed by E29 in TacT2 ${ }_{\mathrm{STm}}$ (Fig. $4 \mathrm{c}$ red and blue structures, respectively). Interestingly, the structure of $\mathrm{TacT}^{17}$ reveals similar stabilizing bonds (K31 with Phe19 and Ala17-Fig. 4c, grey structure). Accordingly, a K31E substitution abolished toxicity of TacT in Salmonella (Fig. 4c, right panel), highlighting the importance of this region in the function of both of these enzymes. TacT3 does not contain a lysine in the corresponding region, but a Gln32 that engages in stabilizing hydrogen bonds with the side chain of the neighbouring Arg17 (Fig. 4c, orange structure). We hypothesized that in the absence of these stabilizing hydrogen bonds, the positively charged groove, leading to the active site of the toxin might be less accessible to the substrate aa-tRNA. We therefore used His-tagged, catalytically inactive TacT2 isoforms as potential substrate-trapping mutants, and tested the binding of TacT2 ${ }_{\mathrm{STm}}^{\mathrm{Y} 137 \mathrm{~F}}$ and TacT2 $2_{\mathrm{SEn}}^{\mathrm{Y} 137 \mathrm{~F}}$ to Salmonella aa-tRNA at $\mathrm{pH}$ 7.0. TacT2 $2_{\mathrm{SEn}}$ Y137F pulled down significantly more aa-tRNA than TacT2 $2_{\mathrm{STm}} \mathrm{Y}_{137 \mathrm{~F}}$ (Fig. $4 \mathrm{~d}$ ), thereby reflecting the absence of activity of the Typhimurium isoform at $\mathrm{pH} 7.0$ (Fig. 4b) and explaining the apparent higher toxicity of TacT $2_{\mathrm{SEn}}$ compared to TacT $2_{\mathrm{STm}}$.

The Tac toxins target partially distinct subsets of aa-tRNAs. We next investigated the specificity of activity of the three TacTs towards aa-tRNAs to understand better how they could work 
a
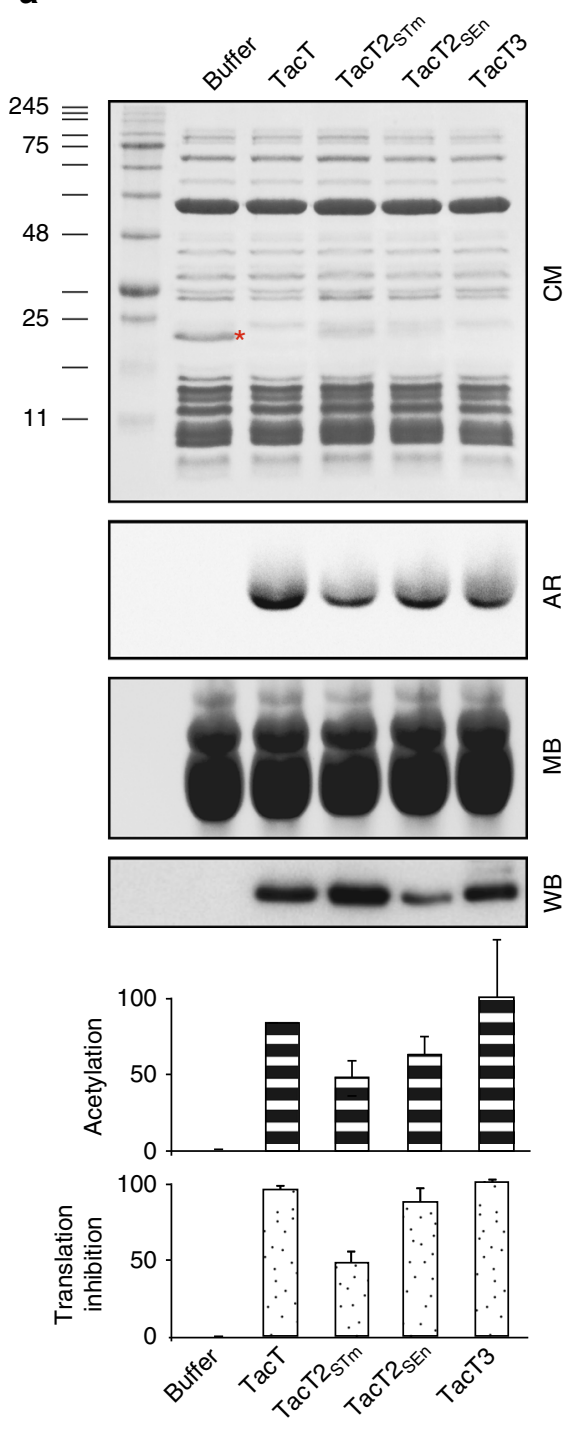

b

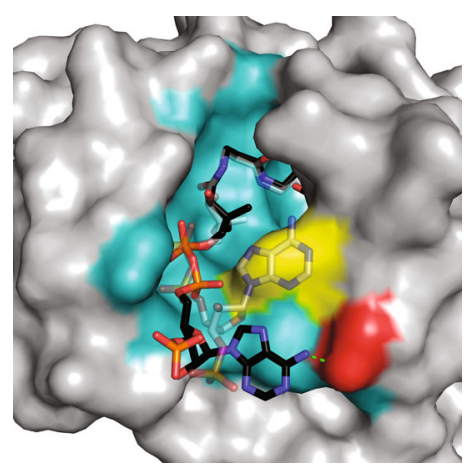

C

C $\frac{\text { TacT }}{-++} \frac{\text { TacT3 }}{-++} \frac{\mathrm{TacT}_{\mathrm{STm}}}{-++} \frac{\mathrm{TacT}_{\mathrm{SEn}}}{-+}$

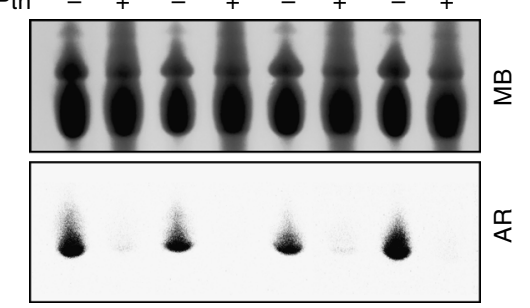

d

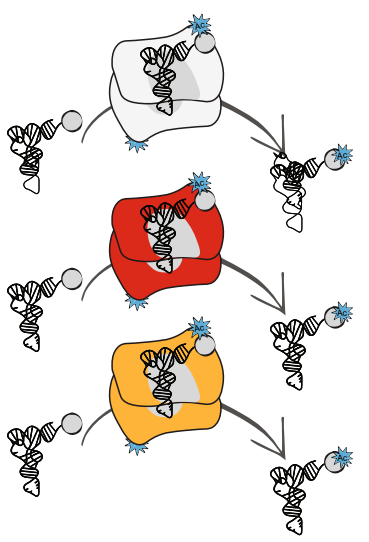

Fig. 3 Tac toxins block translation through acetylation of aminoacyl-tRNAs. a Cell-free expression assays leading to the production of the control protein DHFR (red asterisk) from template DNA without toxins or with purified TacT, TacT2 STm $_{1}$ TacT2 ${ }_{\text {SEn }}$ or TacT3, added from the onset of the assay. $\left.{ }^{14} \mathrm{C}\right]$ AcCoA was added to all the samples. All samples were analysed by SDS-PAGE, and the production of DHFR was revealed by Coomassie staining (CM). tRNAs extracted from the samples were analysed by acid-urea PAGE and revealed by methylene blue staining (MB) and acetylation tracked by autoradiography (AR). Equivalent amount of toxins added to the sample was tested by western blotting against His tag of the toxins (WB). Quantification of inhibition of the production of DHFR (spotted bars) and acetylation of tRNAs (dashed bars) in all conditions is reported in the bar charts, where data represent the mean \pm SEM $(n \geq 3)$. b Surface of the Ac-CoA-binding site of TacT3 with Ac-CoA represented as solid sticks for the orientation in TacT3 and shadowed for the orientation of Ac-CoA in TacT. Trp142 is in yellow and Gly145 is in red. c Exposure of tRNA molecules acetylated by TacTs to Pth treatment in vitro. tRNA molecules acetylated by different TacTs in vitro were subsequently incubated with purified Pth, acetylation was assessed by autoradiography, before or after samples were treated with purified Pth. All the samples were supplemented with $\left[{ }^{14} \mathrm{C}\right] \mathrm{Ac}-\mathrm{CoA}$. Treated tRNA molecules were separated on acid-urea polyacrylamide gel and revealed by methylene blue staining (MB) (top panel). Acetylation was tracked by autoradiography (AR) (lower panel). d Model of activity of Tac toxins

nonredundantly in persister formation. We added purified TacT, TacT2 $2_{\text {SEn }}$, TacT2 $2_{\text {STm }}$ or TacT3 toxin supplemented with Ac-CoA to cell-free expression assays and extracted the tRNA molecules from the samples to obtain acetylated aa-tRNAs among all RNA molecules. Then, relying on the ability of Pth to hydrolyze the ester bond between the acetylated amino acids and the tRNAs, we incubated the samples with purified Pth to liberate the corrupted amino acids. The amino acids were then detected by LC-MS (Fig. 5). The results indicated that following exposure to TacT, 13 different species of amino acids were detected as acetylated
(Supplementary Fig. 10) with a strong bias towards Gly and Ile/ Leu for all four toxins (Fig. 5 and Supplementary Fig. 10). We recovered different amounts of acetylated species for the four toxins (Fig. 5) that matched the extent of their acetylation (Fig. 3a). We investigated whether usage of specific aa-tRNAs by the translation machinery to synthesize DHFR in the cell-free translation assays introduced a bias in the acetylation preference of the toxins. Hence, we repeated the assay with or without the addition of the DHFR template in the samples. For each toxin, the repertoire of the acetylated species was identical in the presence 
a
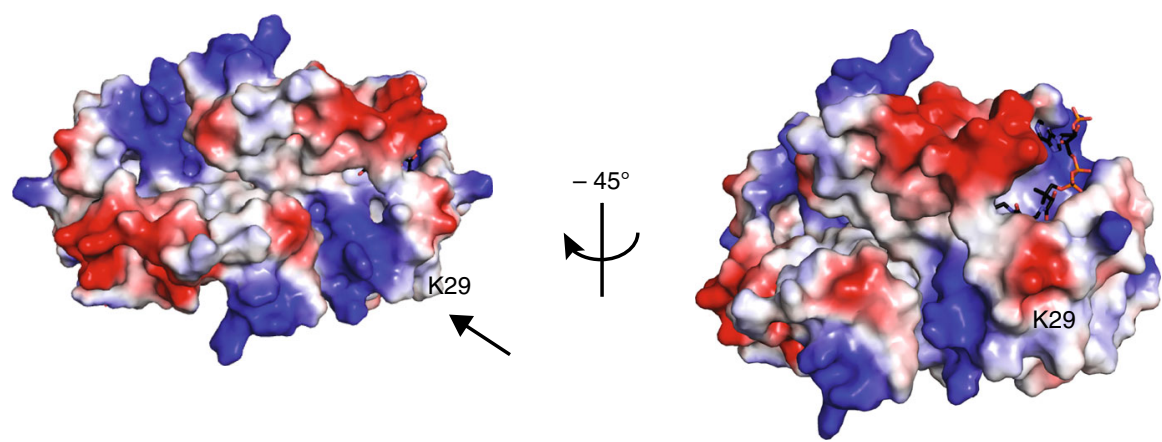

b
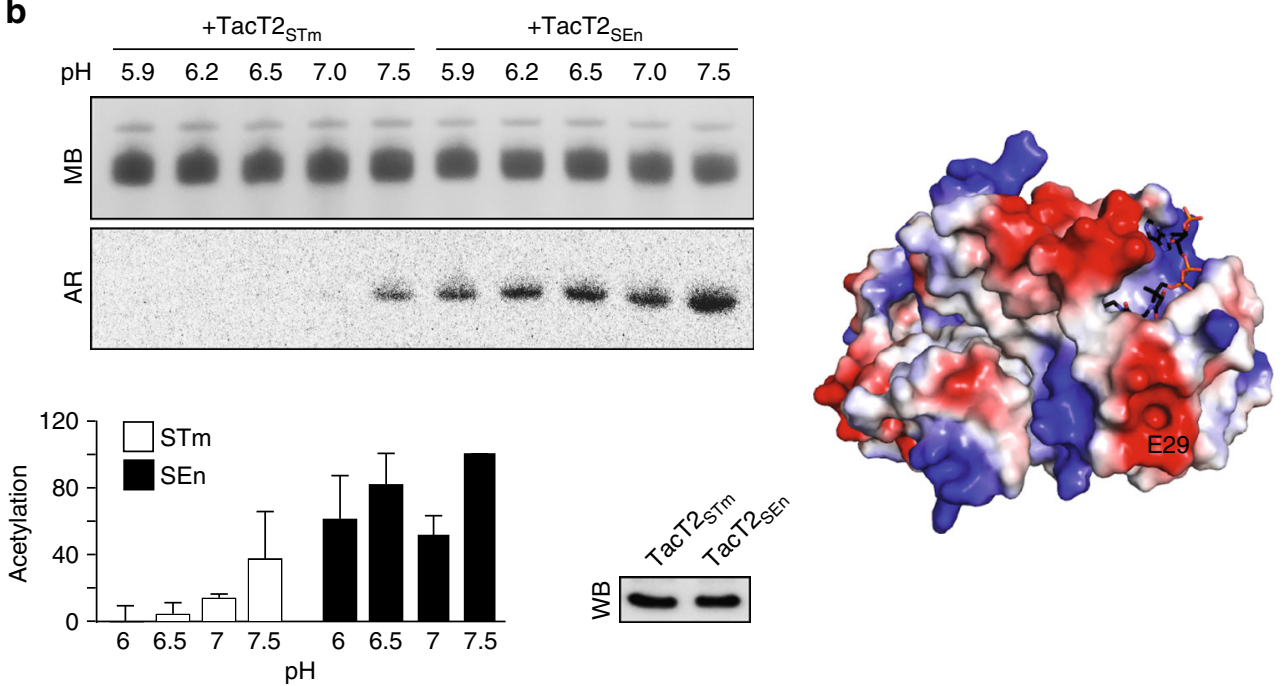

c
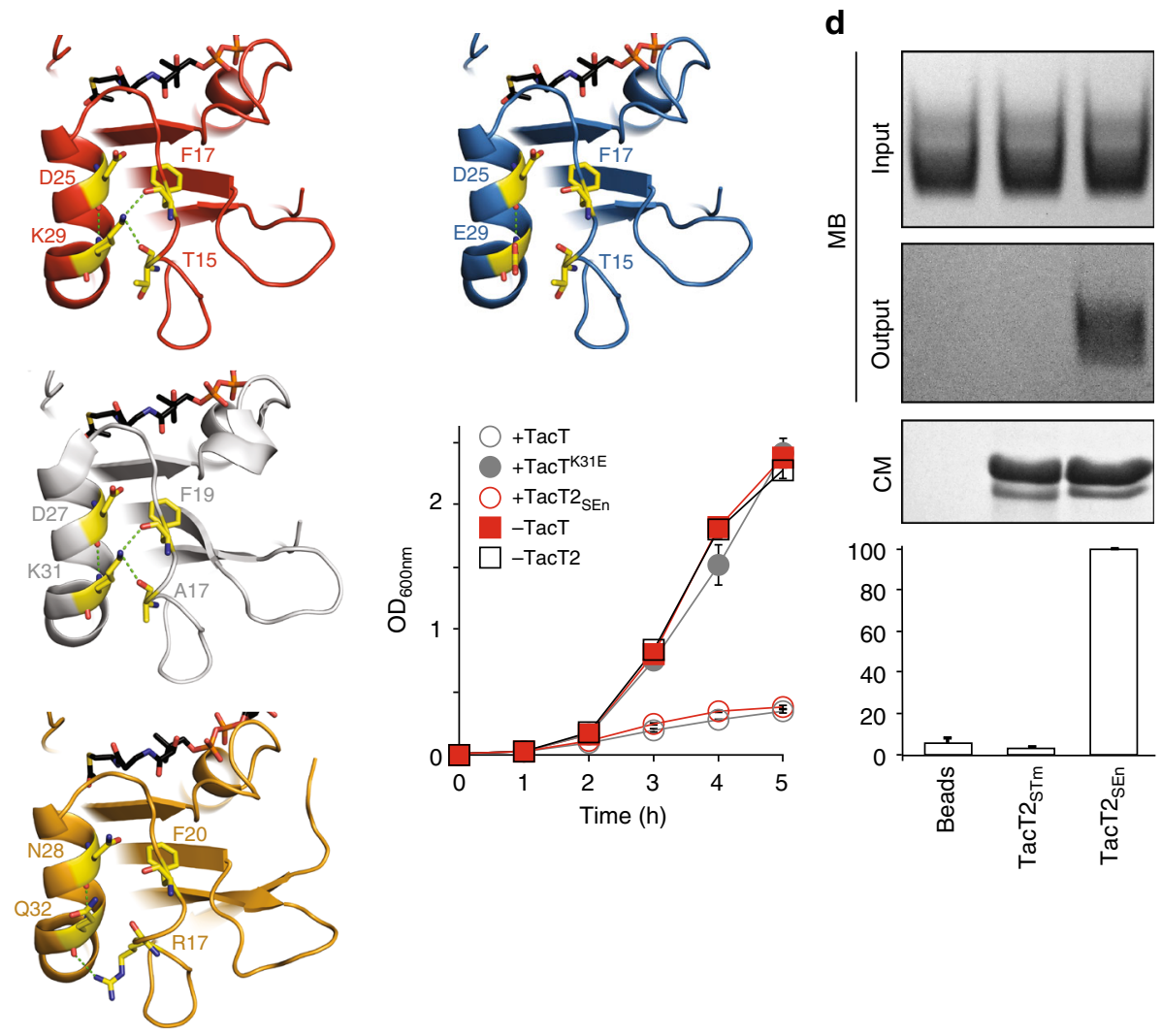
Fig. $4 \mathrm{TaCT} 2_{\mathrm{SEn}}$ acetylates aminoacyl-tRNAs more efficiently than TacT2 $\mathrm{STm}$. a TacT2 polymorphism. Electrostatic potential of the surface of the modelled TacT2 $2_{\mathrm{SEn}}$ (top panels) or TacT2 $\mathrm{STm}$ (lower panel) dimer showing positive potential in blue and negative in red. Arrow points toward the polymorphic amino acid. A $45^{\circ}$ rotation about the $y$-axis from the first view of TacT2 $2_{S E n}$ showing the negative electrostatic potential of E29 in TacT2 2 STm. $\mathbf{b}$ Extracted tRNA molecules from S. Typhimurium $12023 \Delta$ tacATtacAT2tacAT3 were treated with equivalent amounts of TacT2 $2_{\text {STm }}$ or TacT2 2 SEn as assessed by western blotting. $\left[{ }^{14} \mathrm{C}\right] \mathrm{Ac}-\mathrm{CoA}$ was added to all samples from the onset of the assay and the $\mathrm{pH}$ of the reactions was set over a 5.9-7.5 range. Samples were analysed by acid-urea PAGE, tRNAs revealed by methylene blue staining (MB) (top panel) and acetylation tracked by autoradiography (AR) (lower panel). Quantification of acetylation of tRNAs in all conditions is reported in the bar chart, where data represent the mean \pm SEM $(n=3)$. c TacT2 SEn $(r e d)$ K29 and TacT (grey) K31 form two hydrogen bonds between the epsilon amine group of the lysine and carbonyl oxygens from peptide bonds on a neighbouring chain. In a model of TacT2 $\mathrm{STm}$ (blue), no hydrogen bond can be formed with E29. In TacT3 (orange), a stabilizing hydrogen bond is formed between Q32 and the side chain of the neighbouring R17. Growth curves of S. Typhimurium $12023 \Delta$ tacAT expressing from pBAD33, tacT (+TacT), point mutant toxin $\left(+\right.$ TacT $\left.^{\mathrm{K} 31 \mathrm{E}}\right)$ or carrying the empty vector $(-\mathrm{TacT})$, or $\Delta$ tacAT2 expressing from pBAD33 the Enteritidis wild-type toxin $\left(+\right.$ TacT2 $\left.{ }_{\mathrm{SEn}}\right)$ or carrying the empty vector ( - TacT2). All cultures were supplemented with arabinose in fresh rich medium during lag phase. Data represent the mean \pm SEM $(n \geq 3)$. $\mathbf{d}$ Binding of the two isoforms of TacT2 to tRNA molecules. Equivalent amounts of purified His-tagged inactive toxins TacT2 $2_{\text {STm }}{ }^{\text {Y137F }}$ and TacT2 $2_{\text {SEn }}{ }^{\text {Y137F }}$ were incubated with tRNA molecules extracted from S. Typhimurium $12023 \Delta$ tacATtacAT2tacAT3 and subsequently pulled down with anti-His antibody. The input and output tRNA molecules were analysed by acid-urea PAGE and revealed by methylene blue staining (MB-top panels). Equivalent pulldown of the two toxin isoforms was tested by SDS-PAGE and Coomassie staining (CM-lower panel). Quantification of the amount of tRNA molecules pulled down by the toxins is reported in the bar chart where data represent the mean $\pm \operatorname{SEM}(n \geq 3)$

or absence of active translation (Supplementary Figs. 10-11 and Supplementary Data 1), suggesting that usage of aa-tRNAs by translation machinery does not favour the activity of the toxins.

Altogether, these results show that the toxins can acetylate several, but not all amino acids, and even though the spectrum of acetylated species overlapped, the toxins differed in their substrate specificity.

\section{Discussion}

This study extends our understanding of toxin-based growth arrest leading to persister formation of Salmonella. First, using clinical isolates, we show that Salmonella responsible for recurrent non-typhoidal invasive disease respond to internalization by primary human macrophages by dramatically increasing the proportion of persisters present in the population (Fig. 1a). The presence of persisters has been thought to contribute to the recalcitrance of many bacterial infections to antibiotic treatments. However, unambiguous evidence has been brought only recently by the study of invasive Salmonella infections by high-resolution single-nucleotide polymorphism (SNP) analysis. These studies led to differentiating reinfections $(22 \%)$ from clonal relapses $(78 \%)$ in the recurrence of infections $(20-43 \%)^{10}$. The absence of accumulation of any SNP in the relapsing clones provided one of the strongest indications for the involvement of persisters in survival of antibiotic treatment and recurrence of the disease ${ }^{10}$. Our experiments here reveal that the formation of antibiotic-tolerant persisters during infection of macrophages is a common feature of several clinical Salmonella strains and confirm that Salmonella have evolved to respond to phagocytosis by promoting bacterial growth arrest with an approximately 1000-fold increase. This non-growing population, formed as part of a stress response from the bacteria confronted with host immune defence cells could constitute a reservoir for relapse of infection, since we previously showed that at least some of these growth-arrested bacteria can later resume growth, for example, upon death of the host cell.

Chromosomal class II TA modules were shown in S. Typhimurium 12023 to contribute to persister formation, and deletions of each of the single modules led to a defect in persister recovery after infection of macrophages ${ }^{11}$. Therefore, it was surprising to observe that $S$. Enteritidis strains, despite all lacking relBE1 and parDE (Fig. 1b), form high levels of persisters in response to internalization by macrophages. We demonstrate that the single amino acid change, E29K, increases the activity of TacT2 towards promoting persister formation. The enhanced potency of TacT2 $2_{\text {SEn }}$ might compensate for the loss of ParE and RelE1 and account for the ability of $S$. Enteritidis isolates to form persisters at a rate similar to that of $S$. Typhimurium.

TacT2 and TacT3 represent new acetyltransferase toxins ${ }^{17,} 20$ that acetylate the primary amine group of aminoacyl-tRNAs. It appears that the mechanism we first described with $\operatorname{TacT}^{17}$ to block translation is shared by other acetyltransferase toxins and might therefore represent a more general mechanism of growth control $^{20}$.

It was surprising that $\mathrm{TacT} 2_{\mathrm{STm}}$ was not toxic upon overexpression in Salmonella (Fig. 2a). Interestingly, the same enzyme has been reported to be toxic under different overexpression conditions ${ }^{21}$. We show here that TacT2 $2_{\mathrm{STm}}$ has not lost the activity, since the purified toxin was shown to partially block cellfree translation and has a functional acetyltransferase activity (Fig. 3a). It is possible that this moderate toxicity might be revealed by much stronger overexpression ${ }^{21}$. This would be in agreement with our encountered difficulties in cloning $t a c T 2_{S T m}$ in high-copy strong expression vectors ${ }^{22}$ without accumulating mutations in the toxin gene.

This work also revealed the specificity of the three different toxins. The other well-characterized acetyltransferase toxin is Ata $^{20}$, which was shown to be extremely specific in acetylating only initiator Met tRNA. Contrary to this, the Tac toxins target several elongator tRNAs and although there is a strong overlap, they have different target specificities, potentially explaining their non-redundancy. It will be interesting to investigate whether the three TacTs present in each strain of Salmonella are all active at the same time in the exact same cells, and if endogenous factors might influence their spectrum of targets in the bacterial cells, further contributing to a lack of redundancy.

\section{Methods}

Bacterial strains and media. The $S$. Typhimurium strains used in this study were wild-type 12023s and its mutant derivatives and clinical strains (Supplementary Tables 1 and 4). The $S$. Enteritidis strains used in this study are listed in Supplementary Tables 1 and 4 . The E. coli expression strain was BL21 (Invitrogen). All strains were grown at $37^{\circ} \mathrm{C}$ in fully aerated rich growth medium (Luria Bertani) or M9 minimal medium, supplemented when appropriate with $100 \mu \mathrm{g} / \mathrm{ml}$ ampicillin, $25 \mu \mathrm{g} / \mathrm{ml}$ chloramphenicol, $50 \mu \mathrm{g} / \mathrm{ml}$ kanamycin, $0.2 \%$ L-arabinose and/or $0.5-1$ $\mathrm{mM}$ IPTG to allow production of recombinant proteins.

Preparation of primary macrophages. Whole-blood samples were taken from human donors with informed consent and were registered, stored and disposed of according to Imperial College Healthcare Tissue Bank Policy. The authority granted by the Research Ethics Committee to the Tissue Bank to hold a Human Tissue licence and approve work at Imperial using human tissue ensures that the use of human samples is covered by college-wide ethical approval and the Human Tissue Act, 2004. Samples were taken using aseptic technique into sodium heparin 
a

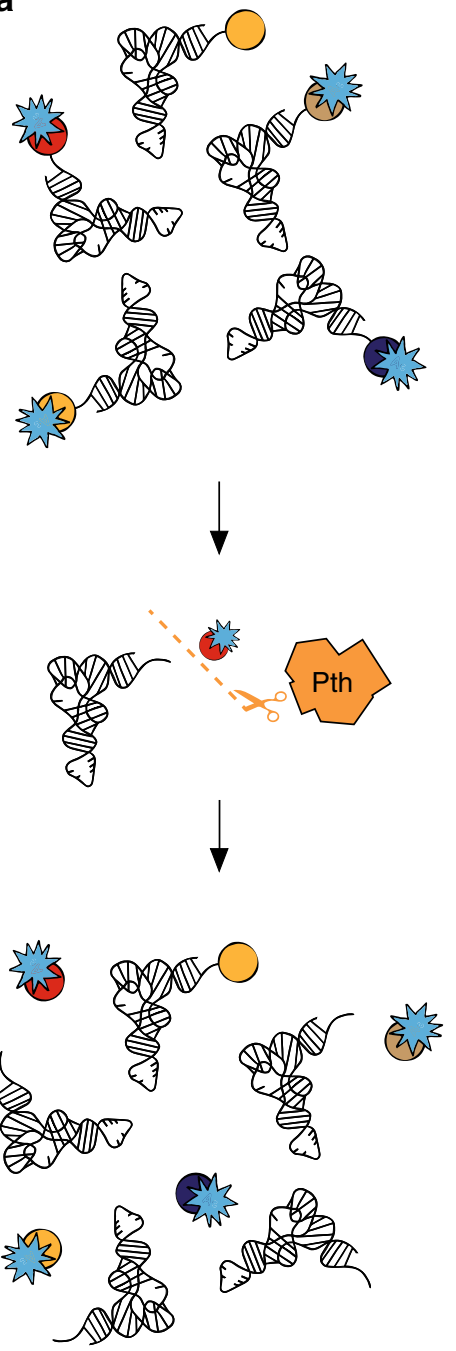

b

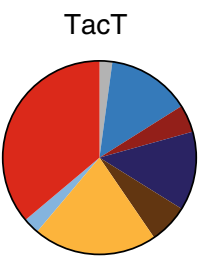

$\mathrm{TacT}_{\mathrm{STm}}$

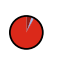

TacT2 SEn

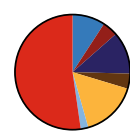

TacT3

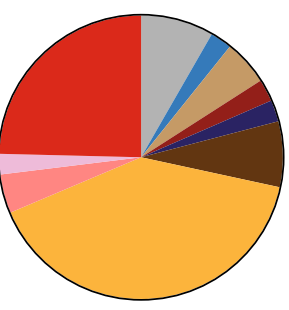

Key

Glycine

Isoleucine/leucine

Tryptophan

Cysteine

Glutamine

Serine

Phenylalanine

Valine

Alanine

Asparagine

Tyrosine

\section{Amino acids}

Fig. 5 The three Tac toxins promote acetylation of specific subsets of aatRNAs identification of amino acids acetylated by the different toxins. a Toxin-corrupted aminoacyl-tRNAs were extracted from cell-free expression assays, and treated with purified Pth to release the acetylated amino acids from tRNAs and subsequently analysed by LC-MS. b Specificity of TacT, TacT2 $2_{\text {SEn }}, T_{a c T} 2_{S T m}$ and TacT3. Size of pie represents the relative amounts of acetylated amino acids recovered

BD vacutainers. Blood samples were centrifuged at $550 \times g$ for $45 \mathrm{~min}$ at room temperature. The top plasma layer was removed and the blood pellet was resuspended in PBS before layering slowly on $20 \mathrm{ml}$ pre-settled Histopaque-1077. The layered samples were then centrifuged at $450 \times g$ for $45 \mathrm{~min}$ at RT. The top layer was removed and the peripheral blood mononuclear cells (PBMC) layer harvested using a Pasteur pipette. The PBMCs were washed three times with $45 \mathrm{ml}$ of prewarmed RPMI and the resulting red blood cell pellet was lysed at $37^{\circ} \mathrm{C}$ in $10 \mathrm{ml}$ lysis buffer $\left(1.5 \mathrm{M} \mathrm{NH}_{4} \mathrm{Cl}, 0.1 \mathrm{M} \mathrm{NaHCO}_{3}, 0.01 \mathrm{M}\right.$ disodium EDTA $\left.0.01 \mathrm{M}\right)$. The remaining cells were pelleted and resuspended in a suitable volume of pre-warmed

RPMI. Primary monocytes were isolated from PBMCs, working swiftly, on ice and using pre-cooled solutions. Cells were passed through a $30 \mu \mathrm{M}$ nylon mesh, and 20 $\mu \mathrm{l}$ of CD14 beads (MACS, Miltenyi Biotec) were added to the samples of $10^{7}$ cells in $80 \mu \mathrm{l}$ of degassed buffer (PBS, 0.5\% BSA, $2 \mathrm{mM}$ EDTA) and incubated for 15 min. Cells were washed in $2 \mathrm{ml}$ buffer and centrifuged at $300 \times g$ for $10 \mathrm{~min}$. The supernatant was aspirated and the pellet was resuspended in $50 \mu$ buffer. CD14+ monocytes were enriched over an MACS column, as per the manufacturer's instructions. Monocytes were resuspended in RPMI-1640 (30 ml per 3-5 $\times 10^{7}$ cells) with $5 \mathrm{ml} \mathrm{L-glutamate}$ and $10 \%$ autologous human serum. Autologous serum was prepared by allowing the whole-blood samples to clot at room temperature for $45 \mathrm{~min}$, followed by centrifugation and collection of the serum.

Rates of protein synthesis. Cells were grown at $37^{\circ} \mathrm{C}$ in LB to stationary phase overnight. Cultures were then diluted to $\mathrm{OD}_{600}$ of 0.1 into fresh $\mathrm{LB}$ medium supplemented with $10 \mathrm{mM}$ IPTG or chloramphenicol $(30 \mu \mathrm{g} / \mathrm{ml}$ to inhibit protein synthesis) and were incubated at $37^{\circ} \mathrm{C}$ with aeration. At 5, 30 and $60 \mathrm{~min}, 500 \mu \mathrm{l}$ samples were harvested, normalized to an $\mathrm{OD}_{600}$ of 0.1 and incubated at room temperature, with $1 \mu \mathrm{Ci}$ of methionine- ${ }^{35} \mathrm{~S}$. After $5 \mathrm{~min}$ of incorporation of radiolabelled isotope, the samples were chased for $10 \mathrm{~min}$ with $0.5 \mathrm{mg}$ of the cold isotope. Cells were then washed with $500 \mu \mathrm{l} 70 \%$ ethanol three times. Pellets were then resuspended in $10 \mu \mathrm{l} 70 \%$ ethanol, dotted onto Whattman paper and exposed to a photostimulable phosphor (PSP) plate overnight. Amounts of incorporated radioactivity were then visualized using a phosphorimager and quantified with ImageJ.

Expression and purification of recombinant $\operatorname{TacT}^{\mathrm{Y} 137 \mathrm{~F}}$ toxins. Salmonella $\mathrm{TacT} 2_{\mathrm{SEn}}{ }^{\mathrm{Y} 137 \mathrm{~F}}$ and $\mathrm{TacT} 2_{\mathrm{STm}}{ }^{\mathrm{Y} 137 \mathrm{~F}}$ sequences encoding the residues were expressed as a N-terminal (His)7 fusion protein of the pQlinkH::TacT2 $2^{\mathrm{Y} 137 \mathrm{~F}}$ vector. E. coli, strain $\mathrm{PC} 2^{23}$, was grown in $\mathrm{LB}$ at $37^{\circ} \mathrm{C}$ until an $\mathrm{OD}_{600}$ of 0.8 was reached, then protein expression was induced overnight at $18^{\circ} \mathrm{C}$ by addition of $0.5 \mathrm{mM}$ IPTG. Cells were lysed by sonication in the lysis buffer $(50 \mathrm{mM}$ Tris $\mathrm{pH} 7.5,500 \mathrm{mM}$ $\mathrm{NaCl}, 1 \mathrm{mM}$ phenylmethanesulfonyl fluoride (PMSF)). Cleared lysate was incubated with Ni-NTA resin (Thermo Fisher) for $2 \mathrm{~h}$ at $4{ }^{\circ} \mathrm{C}$ with agitation. The lysate/ resin mixture was applied to a column and washed with wash buffer $(50 \mathrm{mM}$ Tris $\mathrm{pH} 7.5,500 \mathrm{mM} \mathrm{NaCl}, 20 \mathrm{mM}$ imidazole) and the protein was eluted in $10 \mathrm{ml}$ elution buffer (50 mM Tris $\mathrm{pH} 7.5,500 \mathrm{mM} \mathrm{NaCl}, 500 \mathrm{mM}$ imidazole) and subjected to dialysis (50 $\mathrm{mM}$ Tris $\mathrm{pH} 7.5,500 \mathrm{mM} \mathrm{NaCl})$.

Analysis of tRNA by gel electrophoresis. Total RNA was extracted from Salmonella under acidic conditions to maintain the ester link between tRNA and amino acid/peptide, as described in ref. ${ }^{24}$. This allows isolation of aminoacyltRNAs molecules ${ }^{25}$. aa-tRNA were separated by acid-urea PAGE, as described in ref. ${ }^{25}$ and stained by methylene blue $(500 \mathrm{mM}$ sodium acetate, $0.06 \%$ methylene blue).

Pth purification and in vitro functional assay. Pth was purified as reported in ref. ${ }^{17}$. The activity of Pth was assessed, as previously reported in ref. ${ }^{26}$. Briefly, purified modified tRNAs were incubated with purified Pth $(4 \mu \mathrm{g} / \mathrm{ml})$ in activity assay buffer $(10 \mathrm{mM}$ Tris acetate, $10 \mathrm{mM}$ magnesium acetate, $20 \mathrm{mM}$ ammonium acetate $\mathrm{pH} 8.0) 1 \mathrm{~h}$ at $37^{\circ} \mathrm{C}$.

Expression and purification of recombinant functional toxins. Salmonella TacT, TacT2 $2_{\text {SEn }}$, TacT2 $2_{\text {STm }}$ or TacT3 (with an N-terminal (His)6 purification tag) and cognate antitoxin sequence encoding residues were expressed from the dual expression vector pRSFduet. E. coli $\mathrm{PC} 2$ strain was grown in LB at $37^{\circ} \mathrm{C}$ until an $\mathrm{OD}_{600}$ of 0.8 was reached, then protein expression was induced overnight at $18^{\circ} \mathrm{C}$ by addition of $0.5 \mathrm{mM}$ IPTG. Cells were lysed by sonication in lysis buffer $(50 \mathrm{mM}$ Tris $\mathrm{pH} 7.5,500 \mathrm{mM} \mathrm{NaCl}, 0.5 \mathrm{mM}$ PMSF). Cleared lysate was incubated with NiNTA resin (Qiagen) for $1 \mathrm{~h}$ at $4{ }^{\circ} \mathrm{C}$ with agitation. The lysate/resin mixture was applied to a column and washed with wash buffer $(50 \mathrm{mM}$ Tris $\mathrm{pH}$ 7.5, $500 \mathrm{mM}$ $\mathrm{NaCl}, 20 \mathrm{mM}$ imidazole).

The TA complex bound to the column was denatured with $50 \mathrm{ml}$ denaturation buffer ( $50 \mathrm{mM}$ Tris pH 7.5, $500 \mathrm{mM} \mathrm{NaCl}, 5 \mathrm{M} \mathrm{GnHCl}$ ) and then $50 \mathrm{ml}$ of denaturation wash buffer (50 mM Tris pH 7.5, $500 \mathrm{mM} \mathrm{NaCl}, 5 \mathrm{M} \mathrm{GnHCl}, 20 \mathrm{mM}$ Imidazole). Finally, the denatured toxin was eluted in $10 \mathrm{ml}$ denaturation elution buffer (50 mM Tris pH 7.5, $500 \mathrm{mM} \mathrm{NaCl}, 5 \mathrm{M} \mathrm{GnHCl}, 500 \mathrm{mM}$ Imidazole). The denatured protein was refolded via overnight dialysis at $4{ }^{\circ} \mathrm{C}$ in 21 dialysis buffer (25 mM Tris pH 7.5, $25 \mathrm{mM} \mathrm{NaCl}$, 5\% glycerol) using 3500 MWCO dialysis tubing (Spectrum labs). Refolded TacT1-3 was concentrated and stored at $-80^{\circ} \mathrm{C}$.

Cell-free expression assay. In vitro cell-free expression assays were carried out using the PURExpress In Vitro Protein Synthesis Kit (New England Biolabs [NEB] Massachusetts, USA), following the manufacturer's guidelines, with the exception of the addition of purified toxins ( $2 \mathrm{ng})$ supplemented or not with Ac-CoA ( $2 \mathrm{mM}$ -Sigma-Aldrich) or ${ }^{14} \mathrm{C}$ Ac-CoA $(0.2 \mathrm{mM}, 3.7 \mathrm{mBq}$-Perkin Elmer, Massachusetts, USA) where specified. Samples were analysed by SDS-PAGE or by acid/urea PAGE and visualized by Coomassie Blue staining or by autoradiography. 
Persister assays. Bacterial strains were grown to stationary phase in M9 minimal medium overnight, then diluted to an $\mathrm{OD}_{600}$ of 0.05 into fresh $\mathrm{M} 9$ minimal medium, supplemented with antibiotics as appropriate. $1 \mathrm{mM} \mathrm{IPTG}$ and $0.2 \% \mathrm{~L}$ arabinose was used to induce expression of genes from pCA24N and pBAD33 vectors, respectively, and cultures were incubated at $37^{\circ} \mathrm{C}$. One hour after induction, samples were taken and CFU enumerated $(t=0 \mathrm{~h})$. Cefotaxime $(100 \mu \mathrm{g} / \mathrm{ml})$ was added to the medium and cultures were incubated for $4 \mathrm{~h}$ at $37^{\circ} \mathrm{C}$. After the cefotaxime treatment, $1 \mathrm{ml}$ samples were collected and the surviving bacteria enumerated after the antibiotic was washed out $(t=4 \mathrm{~h})$.

Macrophage-induced persisters were recovered as follows: after $30 \mathrm{~min}$ infection of primary human macrophages with stationary phase bacteria grown overnight in $\mathrm{LB}^{27}$, macrophages were lysed and intracellular bacteria collected, washed once in PBS and resuspended in fresh LB containing various antibiotics. The fold increase in persisters caused by internalization in macrophages was quantified by calculating the ratio of percentage of survival of the macrophageexposed population over that of the same population used for the macrophage inoculum, subsequently grown only in LB containing antibiotics (LB persisters).

Crystallization and structure determination of TacT3 ${ }^{\mathrm{Y} 143 F}$. The purified Tac$\mathrm{T} 3{ }^{\mathrm{Y} 143 \mathrm{~F}}$ proteins $(40 \mathrm{mg} / \mathrm{ml})$ crystallized by sitting drop vapour diffusion against a reservoir of 10\% PEG 6000, $100 \mathrm{mM}$ Bicine $\mathrm{NaOH}$ pH 9.0. Crystals were harvested and submerged in a crystallization buffer containing $20 \%$ glycerol for $10 \mathrm{~s}$ followed by freezing by immersion into liquid nitrogen. Diffraction data were acquired at diamond light source (Oxfordshire, UK) on beamline I04-1. High-resolution native data sets were auto-integrated using autoPROC software ${ }^{28}$. Phases was obtained with PHENIX Phaser software using molecular replacement function and a polyalanine scaffold of TacT (PDB 5FVJ) as a model ${ }^{29}$. The model was then modified using AutoBuild ${ }^{30}$ and manually improved using Coot $^{31}$ and refined using PHENIX and Refmac ${ }^{32,33}$ against the high-resolution native data to give a final structure with an $R_{\text {free }}$ of $23.1 \%$ and good geometry (Supplementary Table 2). The relatively high final $R$ factor could be explained by the flexible loop in the $75-82$ region in one of the monomer units.

Pulldown assay. TacT2 $2_{\mathrm{SEn}}{ }^{\mathrm{Y} 137 \mathrm{~F}}$ or $\mathrm{TacT}_{\mathrm{STm}}{ }^{\mathrm{Y} 137 \mathrm{~F}}(200 \mu \mathrm{g})$ were immobilized on $25 \mu \mathrm{l}$ of Ni-NTA resin (Pierce) followed by extensive washing with buffer A (100 $\mathrm{mM}$ Tris $\mathrm{pH} 8,150 \mathrm{mM}$ sodium chloride). The beads were then equilibrated in buffer B (100 mM HEPES pH 7.5, $150 \mathrm{mM}$ potassium chloride, $5 \mathrm{mM}$ magnesium sulphate, $10 \mathrm{mM}$ ammonium acetate) and incubated with agitation overnight at $4^{\circ}$ $\mathrm{C}$ in the presence of $100 \mu \mathrm{g}$ of total RNA purified from Salmonella Typhimurium $12023 \Delta$ tacATtacAT2tacAT3. After extensive washing with buffer B, the beads were split 1:9. $10 \%$ of total beads was boiled with protein sample buffer followed by SDSPAGE and Coomasie Blue staining, the remaining $90 \%$ was subjected to tRNA extraction under acidic conditions as described previously ${ }^{24}$. The resulting tRNA was separated by acid-urea PAGE as described in ref. ${ }^{25}$ and stained by methylene blue ( $500 \mathrm{mM}$ sodium acetate, $0.06 \%$ methylene blue).

Differential scanning fluorescence (DSF) assay. TacT2 $2_{\mathrm{SEn}}{ }^{\mathrm{Y}} 137 \mathrm{~F}$ or $\mathrm{TacT} 2_{\mathrm{STm}}{ }^{\mathrm{Y}} 137 \mathrm{~F}$ variants were diluted in 96-well plates to a concentration of $10 \mu \mathrm{M}$ in a range of buffers (100 mM of MES pH 5.0, MES pH 6.0, HEPES pH 7.0, sodium phosphate $\mathrm{pH} 7.4$, sodium phosphate $\mathrm{pH} 8.0$, or CHES pH 9.0) all containing $500 \mathrm{mM}$ sodium chloride and $10 \times$ SYPRO Orange dye (Sigma) in a total volume of $30 \mu \mathrm{l}$. A step-wise thermal ramp from 25 to $95^{\circ} \mathrm{C}$ was obtained using Mx3005p instrument (Agilent) and melting temperatures $(\mathrm{Tm})$ were calculated according to established protocols ${ }^{34}$.

Detection of acetylated amino acids. Total RNA was extracted from the cell-free expression assay under acidic conditions to maintain the ester link between tRNA and amino acid/peptide as described in ref. ${ }^{17}$. Purified RNA $(10 \mu l)$ was incubated with $25 \mu \mathrm{g}$ purified Pth in buffer $(10 \mathrm{mM}$ Tris acetate, $10 \mathrm{mM}$ magnesium acetate, $20 \mathrm{mM}$ ammonium acetate $\mathrm{pH}$ 8.) for $1 \mathrm{~h}$ at $37^{\circ} \mathrm{C}$. Samples were combined 1:1:2 with solvent X (40:40:20 acetonitrile:methanol:water) and solvent $\mathrm{Y}(0.2 \%$ acetic acid in acetonitrile), vortexed and precipitate removed via centrifugation. Aqueous normal phase liquid chromatography was performed using an Agilent 1290 Infinity II LC system equipped with a binary pump, temperature-controlled auto-sampler (set at $4{ }^{\circ} \mathrm{C}$ ) and temperature-controlled column compartment (set at $25^{\circ} \mathrm{C}$ ), containing a Cogent Diamond Hydride Type C silica column $(150 \mathrm{~mm} \times 2.1 \mathrm{~mm}$; dead volume $315 \mu \mathrm{l}$ ). A flow-rate of $0.4 \mathrm{ml} / \mathrm{min}$ was used. Elution of polar metabolites was carried out using solvent A consisting of deionized water (resistivity $\sim 18 \mathrm{M} \Omega \mathrm{cm}$ ) containing $0.2 \%$ acetic acid, and solvent B consisting of $0.2 \%$ acetic acid in acetonitrile. The following gradient was used: 0 min $85 \%$ B; $0-2 \min 85 \%$ B; 3-5 min to $80 \% \mathrm{~B}$; 6-7 $\min 75 \% \mathrm{~B} ; 8-9$ min $70 \% \mathrm{~B} ; 10-11 \min 50 \% \mathrm{~B} ; 11.1-14 \mathrm{~min}$ $20 \%$ B; $14.1-25$ min hold $20 \%$ B follow by a 5 min re-equilibration period at $85 \% \mathrm{~B}$ at a flow-rate of $0.4 \mathrm{ml} / \mathrm{min}$. Accurate mass spectrometry was carried out using an Agilent Accurate Mass 6545 QTOF apparatus. Dynamic mass axis calibration was achieved by continuous infusion, post-chromatography, of a reference mass solution using an isocratic pump connected to an ESI ionization source, operated in the positive and negative-ion modes. This instrument enabled accurate mass spectral measurements with an error of less than 5 parts-per-million (ppm), mass resolution ranging from 10,000 to 25,000 over the $\mathrm{m} / \mathrm{z}$ range of $118-955$ atomic mass units, and a 100,000-fold dynamic range with picomolar sensitivity. The data were collected in the centroid $4 \mathrm{GHz}$ (extended dynamic range) mode. Detected $\mathrm{m} / \mathrm{z}$ were deemed to be identified metabolites on the basis of unique accurate massretention time identifiers for masses exhibiting the expected distribution of accompanying isotopomers (Supplementary Table 3). Typical variation in abundance for most of the metabolites stayed between 5 and 10\% under these experimental conditions. Targeted metabolomics was performed using Agilent Mass Hunter Qualitative Analysis B.07.00.

Data availability. The coordinates for the structural model of $\mathrm{TacT}^{\mathrm{Y}}{ }^{\mathrm{Y} 143 \mathrm{~F}}$ have been deposited in the Protein Data Bank under ID code PDB: 6G96.

Received: 8 November 2017 Accepted: 27 April 2018

Published online: 18 May 2018

\section{References}

1. Huson, M. A., Stolp, S. M., van der Poll, T. \& Grobusch, M. P. Communityacquired bacterial bloodstream infections in HIV-infected patients: a systematic review. Clin. Infect. Dis. 58, 79-92 (2014).

2. Reddy, E. A., Shaw, A. V. \& Crump, J. A. Community-acquired bloodstream infections in Africa: a systematic review and meta-analysis. Lancet Infect. Dis. 10, 417-432 (2010).

3. Gordon, M. A. et al. Epidemics of invasive Salmonella enterica serovar enteritidis and S. enterica Serovar typhimurium infection associated with multidrug resistance among adults and children in Malawi. Clin. Infect. Dis. 46, 963-969 (2008)

4. Berkley, J. A. et al. Bacteremia among children admitted to a rural hospital in Kenya. N. Engl. J. Med. 352, 39-47 (2005).

5. Ao, T. T. et al. Global burden of invasive nontyphoidal Salmonella disease, 2010(1). Emerg. Infect. Dis. 21, 941-949 (2015).

6. Chimalizeni, Y., Kawaza, K. \& Molyneux, E. The epidemiology and management of non typhoidal salmonella infections. Adv. Exp. Med. Biol. 659, 33-46 (2010)

7. MacLennan, C. A. \& Levine, M. M. Invasive nontyphoidal Salmonella disease in Africa: current status. Expert. Rev. Anti. Infect. Ther. 11, 443-446 (2013).

8. Feasey, N. A., Dougan, G., Kingsley, R. A., Heyderman, R. S. \& Gordon, M. A Invasive non-typhoidal salmonella disease: an emerging and neglected tropical disease in Africa. Lancet 379, 2489-2499 (2012).

9. Crump, J. A., Sjolund-Karlsson, M., Gordon, M. A. \& Parry, C. M. Epidemiology, clinical presentation, laboratory diagnosis, antimicrobial resistance, and antimicrobial management of invasive Salmonella infections. Clin. Microbiol. Rev. 28, 901-937 (2015).

10. Okoro, C. K. et al. High-resolution single nucleotide polymorphism analysis distinguishes recrudescence and reinfection in recurrent invasive nontyphoidal Salmonella typhimurium disease. Clin. Infect. Dis. 54, 955-963 (2012).

11. Helaine, S. et al. Internalization of Salmonella by macrophages induces formation of nonreplicating persisters. Science 343, 204-208 (2014).

12. Helaine, S. et al. Dynamics of intracellular bacterial replication at the single cell level. Proc. Natl Acad. Sci. USA 107, 3746-3751 (2010).

13. Claudi, B. et al. Phenotypic variation of Salmonella in host tissues delays eradication by antimicrobial chemotherapy. Cell 158, 722-733 (2014).

14. Kaiser, P. et al. Cecum lymph node dendritic cells harbor slow-growing bacteria phenotypically tolerant to antibiotic treatment. PLoS Biol. 12, e1001793 (2014).

15. Hall, A. M., Gollan, B. \& Helaine, S. Toxin-antitoxin systems: reversible toxicity. Curr. Opin. Microbiol. 36, 102-110 (2017).

16. Yamaguchi, Y. \& Inouye, M. Regulation of growth and death in Escherichia coli by toxin-antitoxin systems. Nat. Rev. Microbiol. 9, 779-790 (2011).

17. Cheverton, A. M. et al. A Salmonella toxin promotes persister formation through acetylation of tRNA. Mol. Cell 63, 86-96 (2016).

18. Winther, K. S. \& Gerdes, K. Ectopic production of VapCs from Enterobacteria inhibits translation and trans-activates YoeB mRNA interferase. Mol. Microbiol. 72, 918-930 (2009).

19. Sharma, S. et al. Structural and functional insights into peptidyl-tRNA hydrolase. Biochim. Biophys. Acta 1844, 1279-1288 (2014).

20. Jurenas, D. et al. AtaT blocks translation initiation by $\mathrm{N}$-acetylation of the initiator tRNAfMet. Nat. Chem. Biol. 13, 640-646 (2017).

21. Lobato-Marquez, D., Moreno-Cordoba, I., Figueroa, V., Diaz-Orejas, R. \& Garcia-del Portillo, F. Distinct type I and type II toxin-antitoxin modules control Salmonella lifestyle inside eukaryotic cells. Sci. Rep. 5, 9374 (2015)

22. Scheich, C., Kummel, D., Soumailakakis, D., Heinemann, U. \& Bussow, K. Vectors for co-expression of an unrestricted number of proteins. Nucleic Acids Res. 35, e43 (2007). 
23. Cherepanov, P. LEDGF/p75 interacts with divergent lentiviral integrases and modulates their enzymatic activity in vitro. Nucleic Acids Res. 35, 113-124 (2007).

24. Kohrer, C. \& Rajbhandary, U. L. The many applications of acid urea polyacrylamide gel electrophoresis to studies of tRNAs and aminoacyl-tRNA synthetases. Methods 44, 129-138 (2008).

25. Janssen, B. D., Diner, E. J. \& Hayes, C. S. Analysis of aminoacyl- and peptidyltRNAs by gel electrophoresis. Methods Mol. Biol. 905, 291-309 (2012).

26. Vandavasi, V., Taylor-Creel, K., McFeeters, R. L., Coates, L. \& McFeeters, H. Recombinant production, crystallization and X-ray crystallographic structure determination of peptidyl-tRNA hydrolase from Salmonella typhimurium. Acta Crystallogr. F Struct. Biol. Commun. 70, 872-877 (2014).

27. Figueira, R., Watson, K. G., Holden, D. W. \& Helaine, S. Identification of salmonella pathogenicity island-2 type III secretion system effectors involved in intramacrophage replication of S. enterica serovar typhimurium: implications for rational vaccine design. mBio 4, e00065 (2013).

28. Vonrhein, C. et al. Data processing and analysis with the autoPROC toolbox. Acta Crystallogr. D Biol. Crystallogr. 67, 293-302 (2011).

29. McCoy, A. J. et al. Phaser crystallographic software. J. Appl. Crystallogr. 40, 658-674 (2007).

30. Terwilliger, T. C. et al. Iterative model building, structure refinement and density modification with the PHENIX AutoBuild wizard. Acta Crystallogr. D Biol. Crystallogr. 64, 61-69 (2008).

31. Emsley, P., Lohkamp, B., Scott, W. G. \& Cowtan, K. Features and development of Coot. Acta Crystallogr. D Biol. Crystallogr. 66, 486-501 (2010).

32. Adams, P. D. et al. PHENIX: a comprehensive Python-based system for macromolecular structure solution. Acta Crystallogr. D Biol. Crystallogr. 66, 213-221 (2010).

33. Murshudov, G. N., Vagin, A. A. \& Dodson, E. J. Refinement of macromolecular structures by the maximum-likelihood method. Acta Crystallogr. D Biol. Crystallogr. 53, 240-255 (1997).

34. Niesen, F. H., Berglund, H. \& Vedadi, M. The use of differential scanning fluorimetry to detect ligand interactions that promote protein stability. Nat. Protoc. 2, 2212-2221 (2007).

\section{Acknowledgements}

We thank Prof Cal Maclennan for providing the clinical isolates. We like to thank the manager of the Imperial College London X-Ray crystallography facility, Dr Marc Morgan, for his assistance during protein crystal processing, collection of diffraction data and sharing his structural biology expertize. We are grateful to Agilent Technologies regarding all LC-MS experiments performed on the Agilent 6545 QToF and their support. We thank Dr Severin Ronneau for sharing constructs. We thank members of the Helaine laboratory, Prof David Holden and Dr Vladimir Pelicic for discussions and critical reading of the manuscript. This work was supported by the Medical Research Council (UK) through an MRC Career Development Award MR/M009629/1 to S.H., and MR/J006874/1 to D.W.H. and J.A.R.; MR/J006874/1 to S.H. and A.H.

\section{Author contributions}

Experiments performed by J.A.R., B.G., G.G., A.H., A.M.C. and G.L.M. J.A.R., B.G., G.G., A.H., A.M.C., G.L.M., S.A.H. and S.H. designed experiments and analyzed data. S.H. wrote the paper with input from all other authors.

\section{Additional information}

Supplementary Information accompanies this paper at https://doi.org/10.1038/s41467018-04472-6.

Competing interests: The authors declare no competing interests.

Reprints and permission information is available online at http://npg.nature.com/ reprintsandpermissions/

Publisher's note: Springer Nature remains neutral with regard to jurisdictional claims in published maps and institutional affiliations.

\begin{abstract}
cc) (i) Open Access This article is licensed under a Creative Commons Attribution 4.0 International License, which permits use, sharing, adaptation, distribution and reproduction in any medium or format, as long as you give appropriate credit to the original author(s) and the source, provide a link to the Creative Commons license, and indicate if changes were made. The images or other third party material in this article are included in the article's Creative Commons license, unless indicated otherwise in a credit line to the material. If material is not included in the article's Creative Commons license and your intended use is not permitted by statutory regulation or exceeds the permitted use, you will need to obtain permission directly from the copyright holder. To view a copy of this license, visit http://creativecommons.org/ licenses/by/4.0/.
\end{abstract}

(C) The Author(s) 2018 